\title{
A INOVAÇÃO TECNOLÓGICA NA PERSPECTIVA DOS GESTORES DA INDÚSTRIA MADEIREIRA DA REGIÃO DE LAGES, SC ${ }^{1}$
}

\author{
Flávio José Simioni ${ }^{2}$, Erlaine Binotto ${ }^{3}$ e Debora Nayar Hoff ${ }^{4}$
}

\begin{abstract}
RESUMO - As inovações tecnológicas assumem papel importante na competitividade da indústria madeireira. Assim, o objetivo deste artigo foi descrever como se dá a inovação tecnológica em empresas madeireiras localizadas na Serra catarinense, considerando-se as fontes de inovação tecnológica e as formas de aprendizado que levam à incorporação de tecnologias. Trata-se de uma pesquisa exploratória, utilizando um questionário como instrumento de coleta de dados aplicado a uma amostra de 36 gestores de empresas madeireiras. As categorias de análise foram: as fontes de inovação tecnológias e as formas de aprendizado; os tipos de inovações e o local de aplicação; e as estratégias adotadas para a inovação tecnológica. Os resultados identificaram que a interação com fornecedores a partir de suas próprias potencialidades é a principal forma de buscar a inovação tecnológica nos processos produtivos. As empresas buscam o aprendizado por meio do fazer (learning by doing). Reuniões e capacitações são as formas utilizadas para o estímulo às inovações, que por sua vez são avaliadas no curto prazo. Conclui-se que as inovações tecnológicas são desenvolvidas, sobretudo, por $\mathrm{P} \& \mathrm{D}$ próprios, introdução de máquinas e equipamentos e pelo aprendizado via interação com fornecedores, as quais podem ser classificadas como incrementais, fundamentalmente inseridas no processo produtivo, via estratégias defensivas e imitativas.
\end{abstract}

Palavras-chave: Gestão da Inovação Tecnológica, Indústria de Transformação e Madeira.

\section{TECHNOLOGICAL INNOVATION IN THE PERSPECTIVE OF MANAGERS OF THE LUMBER INDUSTRY IN THE REGION OF LAGES/SC}

\begin{abstract}
Technological innovations take major role in competitiveness of the lumber industry. Thus, the objective of this article was to analyze the management of technological innovation in companies in the mountain chain region in Santa Cantarina. This is an exploratory search, using the questionnaire as a tool applied to collect a sample of 36 timber companies' managers. The categories of analysis are: the sources of innovation learning ways and technologies; the types of innovations and the area of application; and strategies taken to technological innovation. The results identified that the interaction with suppliers from their own potential is the main way of searching technological innovation in the productive processes. Companies seek learning by doing. Meetings and trainings are the forms used for the encouragement to innovations, which in turn are evaluated in the short term. It is concluded that the technological innovations are developed mainly by $P \& D$ themselves, introduction of machinery and equipment and for learning via interaction with suppliers. From this trajectory, the technological innovations are practiced, mainly entered into incremental production process via defensive and imitative strategies.
\end{abstract}

Keywords: Management of Technological Innovation, The Processing Industry and Wood.

\footnotetext{
${ }^{1}$ Recebido em 15.04.2012 aceito para publicação em 04.06.2012.

${ }^{2}$ Universidade do Estado de Santa Catarina. E-mail: <fjsimioni@ hotmail.com>.

${ }^{3}$ Universidade Federal da Grande Dourados, Faculdade de Administração, Ciências Contábeis e Economia. E-mail:<e-binotto@uol.com.br>.

${ }^{4}$ Universidade Federal de Uberlândia, Instituto de Economia. E-mail: <deborahoff@ ie.ufu.br>.
} 


\section{INTRODUÇÃO}

A atividade madeireira da região de Lages, SC, teve seu desenvolvimento inicial baseado na extração da araucária, com duração do início do século XX até o final dos anos 1960. Após esse primeiro ciclo de produção, o setor passou por diversos movimentos de reestruturação, que culminaram na constituição de importantes aglomerações industriais com características semelhantes a um cluster (HOFF; SIMIONI, 2004). A base florestal para as indústrias locais é a produção de madeira do gênero Pinus, a partir da qual se destacam as cadeias produtivas de celulose e papel, madeira sólida e energia.

De acordo com Simioni et al. (2002), a indústria madeireira da região de Lages, SC, se caracteriza, sobretudo, pela produção de madeira serrada proveniente, na sua maioria, de pequenas serrarias de gestão familiar. Outras atividades produtivas que envolvem maior grau de beneficiamento também estavam presentes, como a produção de artefatos, lâminas, painéis e aberturas. A recente organização da cadeia produtiva de energia térmica e elétrica a partir dos resíduos gerados pelas indústrias exemplifica a evolução e diversificação da base produtiva local (SIMIONI; HOEFLICH, 2010).

Hoff e Simioni (2004), entretanto, identificaram que grande parte dos equipamentos utilizados pelas indústrias de processamento primário na região estudada era oriunda do primeiro ciclo da madeira. Essa condição compromete a competividade das empresas e a qualidade da produção, dada a dificuldade para atender aos padrões internacionais, grandes volumes de perdas e resíduos e limitações ao processo inovativo.

Diante desse contexto, as inovações tecnológicas, por permitirem maior dinamicidade competitiva, tendem a assumir papel importante na competitividade da indústria madeireira, sobretudo quando se considera a densidade tecnológica desse setor. Podem ter origem em diferentes fontes, internas e externas à organização, além de assumirem diferentes tipologias de acordo com seu impacto em produtos, processos ou na estrutura organizacional.

Assim, o objetivo deste artigo foi descrever como se dava a inovação tecnológica em empresas madeireiras em Santa Catarina, considerando-se as fontes de informações e as formas de aprendizado que levavam à incorporação de tecnologias.

\section{MATERIAL E MÉTODOS}

A pesquisa teve como área de abrangência as empresas madeireiras situadas na Serra catarinense, contemplando os Municípios de Lages, Capão Alto, Cerro Negro, Anita Garibaldi, Correia Pinto, Otacílio Costa, Campo Belo do Sul e Curitibanos. A população de empresas, conforme dados fornecidos pelo sindicato da categoria e pelas prefeituras, foi d e 95 empresas. De posse desse dado, calculou-se a amostra, seguindo critérios de proporcionalidade por município da região estudada, o que constituiu 36 empresas. Para a coleta de dados, consideraram-se a acessibilidade e disponibilidade dos proprietários ou gerentes do processo produtivo em responder ao questionário. Dessa forma, os sujeitos pesquisados foram os proprietários das empresas (14 deles), denominados proprietários, sócios ou donos; e 22 denominados gerentes administrativos, de produção, de recursos humanos, coordenador de qualidade ou somente gerente. Quanto à escolaridade dos entrevistados, $61 \%$ possuíam formação de nível superior. Todavia, ainda $14 \%$ tinham apenas o ensino fundamental completo.

Na sua maioria, 19 empresas atuavam no desdobro primário da madeira, produzindo madeira serrada, e sete produziam madeira beneficiada. Constituiu ainda a amostra estudada um segundo grupo, composto por empresas que trabalhavam com artefatos de madeira (3), laminação (3), compensados (2), portas (1) e madeira tratada (1). Dois terços das empresas pesquisadas foram classificados como Microempresa (ME) ou Empresa de Pequeno Porte (EPP).

Para esta pesquisa, consideraram-se empresas madeireiras aquelas que atuavam no segmento indústria de transformação primária (desdobro) e secundária (beneficiamento) da cadeia produtiva de madeira sólida, conforme conceito adotado por Simioni et al. (2007). Os instrumentos utilizados na coleta de dados e informações foram um questionário previamente estruturado e as entrevistas, realizadas pelos pesquisadores em 2009. A presença e importância desse segmento na região por longo período de tempo, os ciclos diferenciados na sua maturação e o conhecimento prévio de características setoriais justificam a escolha da região como foco da pesquisa.

Para analisar as fontes de inovações adotadas pelas indústrias, foram considerados o desenvolvimento interno e as fontes externas à empresa. Segundo Tigre 
(2006), o desenvolvimento interno de tecnologias pode ser por meio de atividades de Pesquisa e Desenvolvimento (P\&D), da engenharia reversa ou da experimentação. Como fontes externas, foram examinadas a presença de contratos de transferência de tecnologia e a incorporação de tecnologias através de máquinas e equipamentos.

A aprendizagem organizacional foi analisada, conforme Swieringa e Wierdsma (1995), como mudança do comportamento organizacional, um processo coletivo. Nessa perspectiva, a aprendizagem é entendida como um potencial, uma competência, sendo a mudança vista como demonstração do aprendizado. Para esses autores, a aprendizagem pode ocorrer de modo formal ou informal, via imitação, consciente ou inconsciente, pela repetição.

Para analisar os tipos de inovação, considerou-se a taxonomia utilizada por Freeman e Soete (1997), na qual as mudanças tecnológicas são classificadas em quatro níveis, segundo seus impactos: incremental, radical, novo sistema tecnológico e novo paradigma tecnoeconômico. As mudanças incrementais são aquelas que ocorrem cotidianamente nas empresas, derivadas, sobretudo, do aprendizado interno e da capacitação acumulada. Melhorias feitas nos processos, no design, no layout e na qualidade são exemplos dessas inovações. Já as mudanças consideradas radicais geralmente são derivadas de atividades de $\mathrm{P} \& \mathrm{D}$, rompem a trajetória e dão origem a uma nova rota tecnológica, com saltos de produtividade (TIGRE, 2006). Entretanto, as mudanças que caracterizam um novo sistema tecnológico são mais abrangentes, de modo a afetar mais de um setor econômico e dar origem a novas atividades econômicas. De modo mais amplo ainda, mudanças que alteram toda a economia, seja no aspecto técnico, organizacional ou no social, caracterizam mudança paradigmática.

A avaliação do local onde são incorporadas as inovações tecnológicas foi realizada levando-se em conta o Manual de Oslo (OCDE, 2004) e Tigre (2006). Considera-se inovação de um produto quando ele sofre melhoria significativa, cujas características principais diferem dos já produzidos pela empresa. Já a inovação de processo ocorre quando há a adoção de métodos de produção ou comercialização novos ou significativamente aprimorados, podendo envolver mudanças de equipamentos, recursos humanos, métodos de trabalho ou a combinação deles.
Para avaliar a gestão estratégica relacionada à inovação tecnológica, considerou-se a classificação proposta por Tigre (2006), podendo ser ofensiva, defensiva, imitativa, dependente, tradicional ou oportunista. As escolhas estratégicas estão diretamente relacionadas às fontes de inovação tecnológica utilizadas pelas organizações.

\section{RESULTADOS}

Das 36 empresas pesquisadas, 11 relataram ter realizado alguma inovação tecnológica, representando $31 \%$ da amostra. As principais fontes de inovação tecnológica são apresentadas na Tabela 1. Percebe-se, nessa tabela, que são destacados o $\mathrm{P} \& \mathrm{D}$ próprio, eventos, aprendizado cumulativo, reengenharia, cursos e treinamentos.

As empresas trabalham com a ideia de aprender com o próprio processo, ou seja, executando-se a atividade ou o processo que sofreu mudanças. A interação com clientes e fornecedores é outra forma utilizada para o aprendizado. As duas são complementadas pela busca de informações e dados externos que possam ajudar no entendimento e desempenho da mudança implementada. Via de regra, o conhecimento dessas possibilidades de inovação se dá por meio da participação em feiras especializadas do setor florestal e madeireiro. Os métodos de aprendizagem estão demonstrados na Figura 1.

Para 11 pesquisados, a forma de aprendizado mais importante é o planejamento, momento em que buscam aprender acerca do que foi modificado, executando e avaliando as atividades. Essa forma é adotada pelo aprendizado decorrente da observação de seus próprios erros, para sete deles; pela repetição, para outros sete; pela imitação, para três; e pela observação dos erros cometidos por outras empresas, para dois dos entrevistados. O estímulo ao aprendizado e a geração de novas ideias têm sido adotados pelas empresas a partir da realização de reuniões periódicas, para sete dos pesquisados; reuniões determinadas pela necessidade, para outros sete; treinamentos específicos, para seis deles; e programas de incentivo ou premiações, para sete dos respondentes. A principal forma de inovação tecnológica adotada pelas empresas entrevistadas ocorria através da introdução de máquinas e equipamentos mais modernos e automatizados. Entretanto, mencionaram que as mudanças adotadas eram tipicamente pequenas e

Revista Árvore, Viçosa-MG, v.36, n.5, p.981-987, 2012 
Tabela 1 - Principais fontes de inovação tecnológica utilizada pela indústria madeireira. Table 1 - Main sources of technological innovation used by the lumber industry.

\begin{tabular}{lcc}
\hline Fontes de inovação tecnológica & Número de indicações \\
\hline P\&D Próprio & 8 & 15,7 \\
Eventos: feiras e exposições & 8 & 15,7 \\
Aprendizado cumulativo: ideias dos funcionários & 8 & 5 \\
Reengenharia: reformulação ou realinhamento de atividades ou processos & 5 & 4,7 \\
Cursos e treinamentos & 4 \\
P\&D com fornecedores & 3 \\
Marketing & 3,8 \\
Imitação de outra empresa & 7,8 \\
Bibliografia: livros, revistas, jornais e internet & 5,9 \\
Consultoria ou contratação de RH experientes & 5,9 \\
P\&D com Universidades & 3,9 \\
Transferência via licenças e patentes & 2 \\
Software aplicativo & 3,9 \\
\hline
\end{tabular}

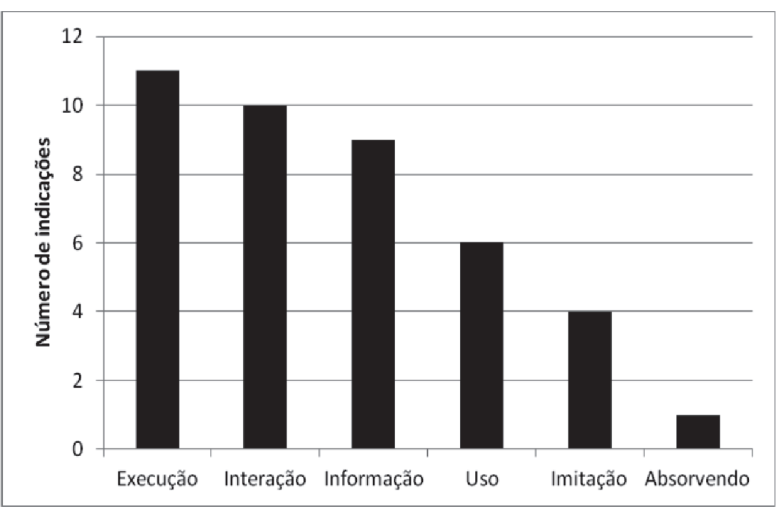

Figura 1 - Principais métodos de aprendizagem utilizados pela indústria madeireira.

Figure 1-Main learning methods used by the lumber industry.

cotidianas. Na Figura 2 são apresentas as principais alterações no processo produtivo apontadas pelas empresas entrevistadas.

Com relação aos resultados obtidos pelas inovações tecnológicas, verificou-se que $80 \%$ das empresas apresentavam alguma estratégia ou metodologia para medi-los. Para tanto, eram utilizados como critérios principais a quantidade produzida, parâmetros de qualidade e indicadores de eficiência no processo produtivo. A periodicidade das avaliações era realizada diariamente em $70 \%$ dos casos, e as demais empresas efetuavam-na semanal ou anualmente, para $10 \%$ e $20 \%$, respectivamente.

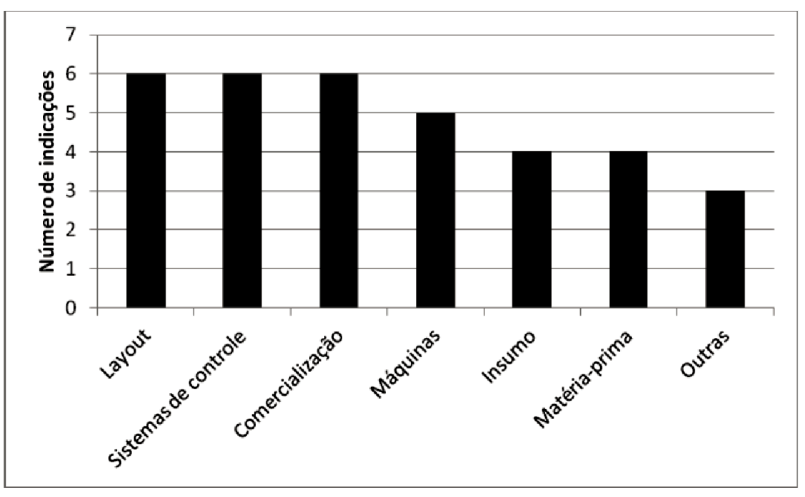

Figura 2 - Principais áreas onde são realizadas as inovações tecnológicas de processo.

Figure 2-Main learning methods used by the lumber industry.

\section{DISCUSSÃO}

A partir das informações apresentadas na Tabela 1 e das constatações obtidas a campo, destaca-se a importância das fontes internas para a inovação tecnológica, caracterizadas pelo conhecimento próprio via $\mathrm{P} \& \mathrm{D}$ (aqui entendido como o ato de testar, avaliar, redimensionar ou ensaiar) e cumulativo, em que o aprendizado é reforçado com a participação em feiras e exposições, cursos e treinamentos. De acordo com Pavitt (1984), esse conhecimento tecnológico se transforma em informação, cuja aplicação é específica, com ação cumulativa no desenvolvimento das firmas. Essa modalidade é relevante pelas características do processo produtivo, que ainda depende muito da ação humana. 
Uma segunda fonte de inovação ocorre através de contratos de transferência de tecnologia que está, na maioria das vezes, associada com a aquisição de máquinas e equipamentos. Acompanham também ações de reengenharia, treinamentos e $\mathrm{P} \& \mathrm{D}$ com fornecedores, de modo a intensificar a absorção de tecnologias externas, possibilitando o aumento da escala de produção, melhoria de qualidade dos produtos e redução de custos, tornando as empresas mais competitivas. Para Tigre (2006), trata-se de oportunidade para obter salto tecnológico nos processos ou produtos e de importante alternativa de aumento da produtividade.

Entre os dados que caracterizam os métodos e as formas de aprendizado adotadas pelas empresas inovadoras, segundo Dosi (1988), há o predomínio do learning by doing através da repetição e experimentação, que se processam na execução das tarefas e nas relações que se formam nos processos produtivos. O learning by interacting ocorre em função das interações com os fornecedores, sobretudo com o segmento de máquinas e equipamentos que são introduzidos no processo produtivo. A imitação e a observação dos erros cometidos por outras empresas, bem como outras informações de origem externa, refletem o learning by imiting aprendizado gerado da reprodução de inovações introduzidas por outras firmas.

Considerando Swieringa e Wierdsma (1995), pode-se inferir que a aprendizagem é mudança de conduta que ocorre informalmente, pela imitação e mediante a experiência ou por meio da ação, e o resultado do que se aprendeu fica evidenciado nas tarefas que são realizadas e, também, de modo inconsciente, através da repetição.

A classificação e seleção de tábuas são exemplos de inovação tecnológica na indústria madeireira que leva a uma mudança de conduta. Essa atividade normalmente é realizada visualmente por classificadores humanos e pode ser substituída utilizando imagens digitais, quando se necessita de um aperfeiçoamento do sistema de identificação de características apropriadas para discriminar os defeitos da madeira (KHOURY JUNIOR et al., 2005).

As empresas estimulam novas ideias e as trocas ou difusão entre seus colaboradores, adotando estratégias que estão fundamentalmente permeadas pelo ambiente organizacional e produtivo de inserção dos indivíduos. É incipiente a adoção de meios sistematizados, da implementação de ações de pesquisa e de parcerias que possam gerar mudanças mais impactantes nos processos e produtos. Observando os mecanismos internos adotados pelas empresas para estimular a difusão das inovações, pode-se verificar que estão centrados no desenvolvimento das habilidades técnicas entre os usuários (learning by using), conforme ponderou Deza (1995).

Limitações de conhecimentos técnicos e específicos foram apontadas por Simioni e Hoeflich (2010) ao realizarem estudos de prospecção no segmento da indústria madeireira, identificando a necessidade urgente de qualificação profissional dos colaboradores, agravada pelo baixo nível de escolaridade.

Considerando o exposto, pelo qual se evidenciaram o aprendizado interno e a capacitação acumulada, corroboradas pelas informações apresentadas na Figura 2, em que se destacam as inovações em layout, sistemas de controle e comercialização e em máquinas e equipamentos do processo produtivo, podem-se classificar as inovações, segundo a taxonomia de Freeman e Soete (1997), como mudanças incrementais e, de acordo com o Manual de Oslo (OCDE, 2004) e Tigre (2006), como mudanças de processo.

As avaliações diárias dos resultados das inovações dão uma visão pontual do processo, não permitindo a observação de mudanças ou tendências ao longo do tempo. Apesar de serem muito indicadas para processos como os que ocorrem no setor de base florestal, precisam ser acompanhadas por outros tipos de avaliação para serem usadas como bons indicadores gerenciais.

Ao analisar a eficiência técnica da indústria de base florestal nos estados brasileiros, Santos (2011) atribuiu os altos custos das operações industriais como principal fonte de ineficiência, chamando a atenção para a necessidade de controle austero dos fatores que compõem seus custos, devendo direcionar maiores esforços para a sua redução.

A partir da descrição da maioria das tecnologias incorporadas pelas empresas pesquisadas, pode-se classificá-las, em seu grau de maturidade, como tecnologias-base (WAACK, 2000), uma vez que são essenciais ao desenvolvimento do processo produtivo, mas não contribuem sobremaneira para o desenvolvimento de competitividade ou de grande diferencial da firma perante seus concorrentes. Complementarmente, nas

Revista Árvore, Viçosa-MG, v.36, n.5, p.981-987, 2012 
empresas que produzem produtos de maior valor agregado e atuam no mercado externo, verificou-se a existência de tecnologias-chave, por apresentarem impacto direto sobre a produtividade e qualidade e, sobretudo, nos custos de produção, com impactos positivos sobre a competitividade, o que era esperado, de acordo com a literatura sobre o tema (COUTINHO; FERRAZ, 1997).

A definição das estratégias competitivas está muito relacionada com o tipo de inovação perseguida pelas empresas, ainda muito vinculado a aspectos pontuais do processo produtivo, no qual se buscam o controle dos custos, o estabelecimento de um padrão de qualidade (muitas vezes relacionado à redução de variabilidade no processo e no produto) e a redução de perdas (que acaba interferindo no custo de produção). Como consequência desse padrão comportamental, tem-se um conjunto de organizações que, conforme Tigre (2006), opta por trabalhar com estratégias defensivas e imitativas.

Esse comportamento guarda estreito relacionamento com o tipo preponderante de empresa no setor estudado: as serrarias. Embora com muitas limitações tecnológicas nos processos produtivos, historicamente essas empresas se mantêm no mercado, o que é usado como argumento para justificar a manutenção do seu status quo. Isso não significa, necessariamente, que o mercado consumidor tenha deixado de ampliar suas exigências e necessidades (demand pull). Por conta disso, algumas empresas sobressaem no contexto geral pelas incorporações de inovações tecnológicas, tornando-as mais competitivas, de modo a incrementar o portfólio de produtos oferecidos e os mercados atendidos. Vale enfatizar que esse é um comportamento esperado do setor, dadas as constatações de pesquisas anteriores feitas sobre esse assunto, como em Simioni et al. (2002) e Hoff e Simioni (2004).

\section{CONCLUSÕES}

Constatou-se que os processos inovativos e de adoção de novas tecnologias caracterizam-se como processos desafiadores para as empresas do setor madeireiro, seja pela sua trajetória tecnológica adotada, seja, também, pelos novos condicionantes da competitividade.

As principais fontes de tecnologias na indústria madeireira originam-se tanto internamente, via aprendizado próprio, quanto externamente, via introdução de máquinas e equipamentos, estabelecendo vínculos com fornecedores. As formas de aprendizado preponderantes decorrem do fazer e da repetição (learning by doing), da interação com os fornecedores (learning by interacting) e da imitação das inovações geradas por outras firmas (learning by imiting). A difusão das inovações nas empresas ocorre a partir do desenvolvimento de habilidades técnicas entre os usuários (learning by using).

Considerando essas características, as inovações tecnológicas praticadas são incrementais, fundamentalmente inseridas no processo produtivo, via estratégias defensivas e imitativas. Percebeu-se, também, que as inovações tendem a ocorrer com mais frequência em processos do que em produtos.

Destaca-se que o estudo é limitado por considerar as empresas de apenas uma região produtiva e que os resultados podem estar viesados pela cultura organizacional desenvolvida ao longo da história e pelo contexto de inserção das firmas. A ampliação do estudo para outras regiões produtoras com características de processo de produção semelhantes àquelas encontradas nas firmas da Serra catarinense é recomendada e pode ser relevante, por permitir a identificação de características gerais.

\section{AGRADECIMENTOS}

À Fundação de Amparo à Pesquisa e Inovação do Estado de Santa Catarina (FAPESC), pelo apoio financeiro; e à Universidade do Planalto Catarinense (UNIPLAC), pelo apoio institucional.

\section{REFERÊNCIAS}

\author{
COUTINHO, L; FERRAZ, J. C. Estudo da \\ competitividade da indústria \\ brasileira. São Paulo: Papirus, 1997.
}

DEZA, X. V. Economía de la innovación y del cambio tecnológico. Madri: Siglo XXI, 1995.

DOSI, G. Sources, procedures and microeconomics effects of innovation. Journal of Economics Literature, v.26, n.3, p.1120-1171, 1988.

FREEMAN, C.; SOETE, L. The economics of industrial innovation. Cambridge: MIT Press, 1997. 
HOFF, D. N.; SIMIONI, F. J. O setor de base florestal na serra catarinense. Lages: Uniplac, 2004. 268p.

KHOURY JUNIOR, J. K. et al. Análise discriminante paramétrica para reconhecimento de defeitos em tábuas de eucalipto utilizando imagens digitais. Revista Árvore, v.29, n.2, p.299-309, 2005.

OCDE. Manual de oslo: proposta de diretrizes para coleta e interpretação de dados sobre inovação tecnológica. Brasilia: OCDE/FINEP, 2004.

PAVITT, K. Sectorial patterns of technical change: towards a taxonomy and a theory. Research Policy, v.13, n.6, p.343-373, 1984.

SANTOS, R. B. N. Eficiência técnica na indústria de base florestal brasileira via metas intermediárias. Revista Árvore, v.35, n.6, p.1319-1326, 2011.

SIMIONI, F. J.; BRAND, M. A.; ROTTA, D. N. H. Caracterização do cluster da madeira da região de Lages/SC. Lages: UNIPLAC, 2002. (Relatório de Pesquisa).
SIMIONI, F. J. et al. Reflexões a respeito do conceito de cadeia produtiva no agronegócio florestal. In: BINOTTO, E. (Org.). Tecnologia e processos agroindustriais. Passo Fundo: Editora da Universidade de Passo Fundo, 2007. p.97-116.

SIMIONI, F. J.; HOEFLICH, V. A. Cadeia produtiva de energia de biomassa na região do Planalto Sul de Santa Catarina: uma abordagem prospectiva. Revista Árvore, v.34, n.6, p.1091-1099, 2010.

SWIERINGA, J.; WIERDSMA, A. La organización que aprende. Wilmington: Addison-Wesley, 1995.

TIGRE, P. Gestão da inovação: a economia da tecnologia no Brasil. Rio de Janeiro: Elsevier, 2006.

WAACK, R. S. Gerenciamento de tecnologia e inovação em sistemas agroindustriais. In: ZYLBERSTAJN, D.; NEVES, M. F. Economia e gestão dos negócios agroalimentares: indústria de alimentos, indústria de insumos, produção agropecuária, distribuição. São Paulo: Pioneira, 2000. p.323-348. 
